\title{
Coronary arterio-venous fistulae
}

\author{
C. P. NEWCOMBE, ${ }^{1}$ W. WHITAKER, AND P. G. KEATES \\ From the Departments of Cardiology and Diagnostic Radiology, General Infirmary, Leeds
}

The discovery of a continuous murmur at an unusual site over the praecordium presents an interesting diagnostic problem. Abnormalities of the coronary circulation are among the possible causes for such a finding, but they are rare.

In this report we describe three patients with coronary arterio-venous fistulae and draw attention to the ease and elegance of the diagnosis of this condition by retrograde aortography.

\section{CASE REPORTS}

CASE 1 (B. H.) A woman aged 38 years was referred for assessment of a murmur first heard at the age of

Present address: York County Hospital
7 years. Slight dyspnoea dated from 'influenza' three? months previously.

She was of average build. The blood pressure was $120 / 75 \mathrm{~mm}$. $\mathrm{Hg}$. She was in sinus rhythm with a normal cardiac impulse, no thrill and normal heart sounds. There was a continuous murmur maximal over the mid-sternum, audible equally to the right $\overrightarrow{-}$ and left of the mid-line; it was not heard in the left infraclavicular fossa, and the systolic component only was heard at the apex. The routine radiological examination revealed no abnormality (Fig. 1).

Conventional right heart catheterization was per- $\vec{\varphi}$ formed together with retrograde aortic catheterization $\stackrel{+}{\perp}$ by the Seldinger method. Pressures were 25/8, 25/0. and $100 / 60 \mathrm{~mm} . \mathrm{Hg}$ respectively in pulmonary artery, right ventricle, and aorta. Blood oxygen saturation values were: superior vena cava, $62 \%$; inferior vena

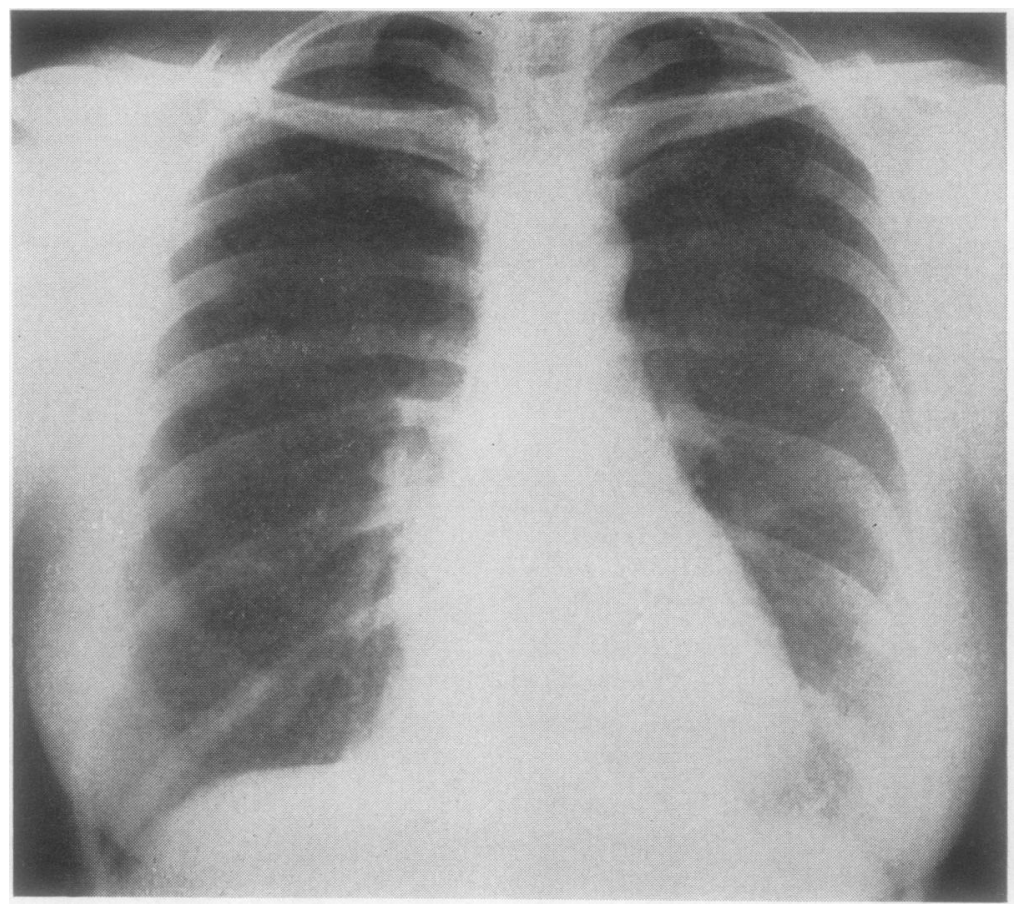

FI(j. 1. Case 1. Chest radiograph shows no abnormality of the heart or lungs. 
cava, $59 \%$; right atrium, $63 \%$; right ventricle inflow, $68 \%$; right ventricle outflow, $67 \%$; pulmonary artery, $68 \%$; and aorta, $95 \%$, indicating a left to right shunt into the right ventricle. A dye curve recorded from the ear after the injection of Coomassie blue into the pulmonary artery confirmed the existence of the small left to right shunt (Fig. 2).

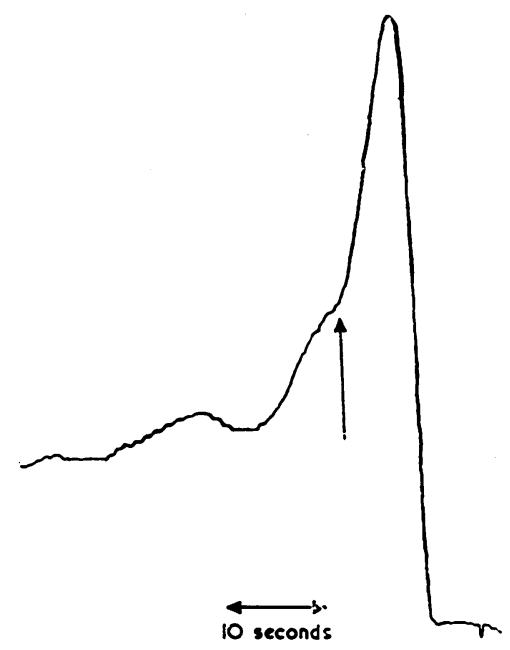

FIG. 2. Case 1. Dye dilution curve recorded from the ear after the injection of Coomassie blue into the pulmonary artery. The appearance-time and up stroke of the curve are normal but the break on the down stroke (arrowed) indicates a small left to right shunt.

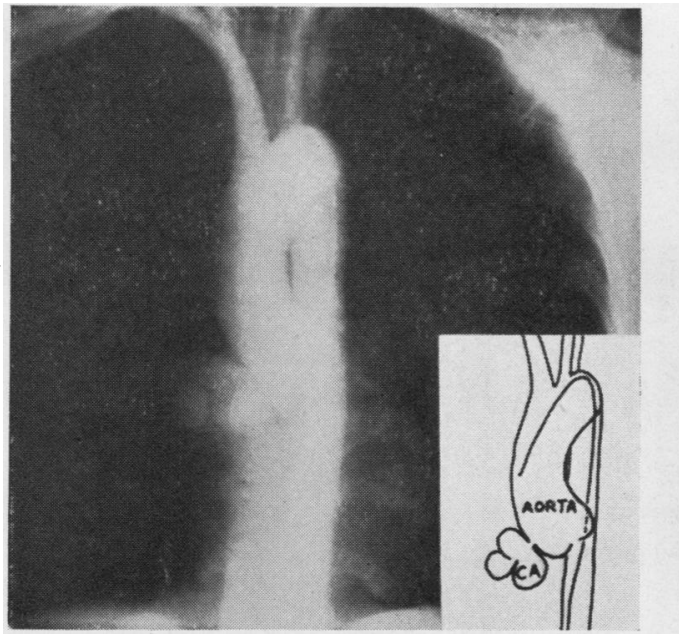

Aortography (Fig. 3) demonstrated a tortuous dilated right coronary artery with slight opacification of the right ventricle, confirming that the aberrant vessel entered this chamber. The left coronary artery was normal.

CASE 2 (S. B.) A symptomless child of 12 years was referred for assessment of a murmur discovered on routine examination when she was aged 5 .

She was obese. There was sinus rhythm with a normal pulse and a blood pressure of $120 / 55 \mathrm{~mm}$. $\mathrm{Hg}$. The apex was in the normal position but there was a slight parasternal lift, suggesting right ventricular enlargement. The first heart sound was normal; splitting of the second sound persisted on expiration though it was less than on full inspiration. There was a loud continuous murmur maximal in the second right intercostal space but no thrill.

The electrocardiogram was normal. On fluoroscopy there was a broad heart shadow with some right ventricular enlargement. The lung fields were normal and there was no hilar dance (Fig. 4).

Right heart and retrograde aortic catheterization were performed as in case 1 . Pressures were $30 / 10$ and $30 / 0 \mathrm{~mm} . \mathrm{Hg}$ in the pulmonary artery and right ventricle. Blood oxygen saturation values were: superior vena cava, $71 \%$; inferior vena cava, $71 \%$; right atrium, $83 \%$; right ventricle inflow, $81 \%$; right ventricle outflow, $82 \%$; pulmonary artery, $81 \%$; and aorta, $95 \%$, indicating a left to right shunt into the right atrium. A dye curve recorded from the ear after the injection of Coomassie blue into the pulmonary artery demonstrated a large left to right shunt (Fig. 5).

Aortography (Figs. 6 and 7) showed that from the left coronary artery there arose normal anterior descending and circumflex branches, but that in

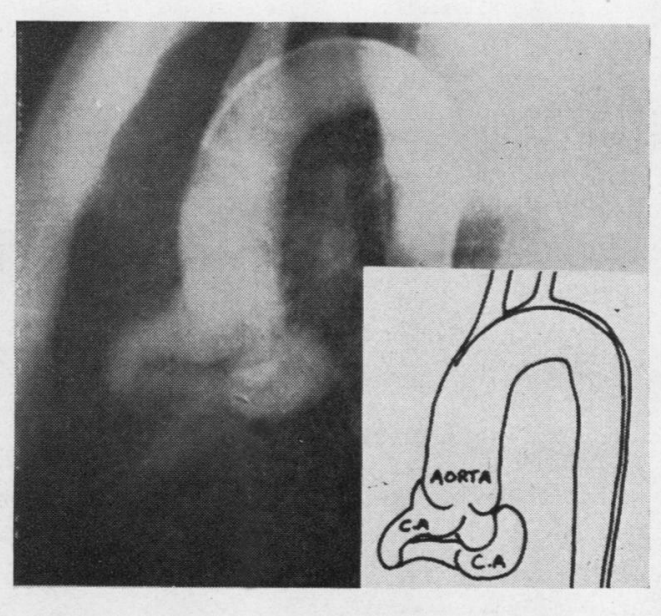

FIG. 3. Case 1. Retrograde thoracic aortogram. Antero-posterior and lateral films. The right coronary artery is much dilated and runs a tortuous course. Slight opacification of the right ventricle indicates a fistulous communication. The left coronary artery is normal. 


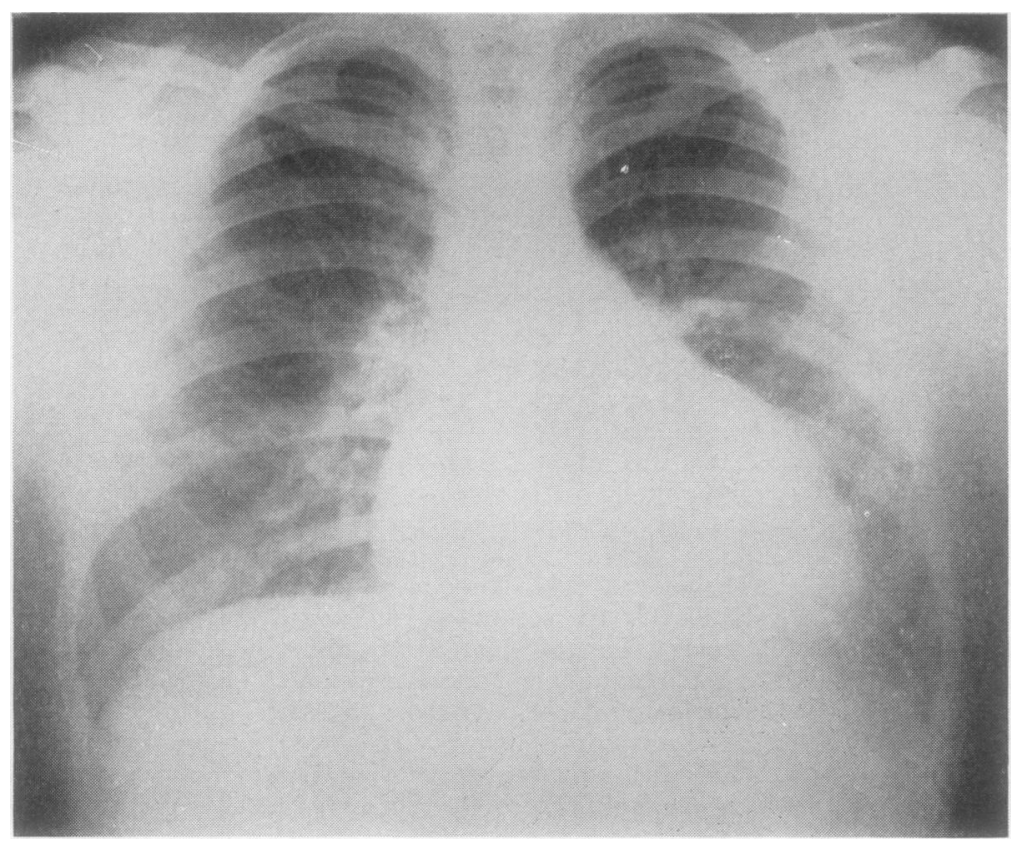

FIG. 4. Case 2. Chest radiograph $\overrightarrow{\overrightarrow{7}}$ shows the heart is enlarged but the? lung fields are within normal limits.

FIG. 6. Case 2. Retrograde thoracic aortogram. Antero-posterior andu lateral films. The left coronary artery" gives rise to normal anterior descend $\overrightarrow{0}$ ing and circumflex branches, but in addition there is a large vossel arising from it and running to the right tof enter the right auricle. Right coronary art sry normal.

FIG. 7. Case 2. Retrograde thoracic $\overrightarrow{\vec{\sigma}}$ aortogram. Lat: film showing opaques medium in the right auricle, right ventricle and pulmonary artery.

FIG. 4

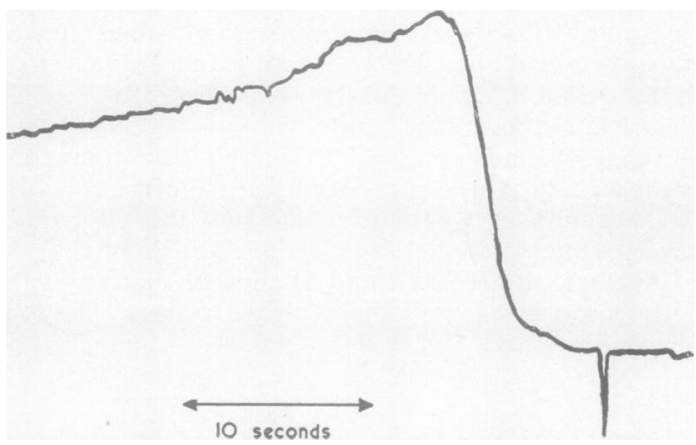

FIG. 5. Case 2. Dye dilution curve recorded from the ear after the injection of Coomassie blue into the pulmonary artery. The appearance time and up stroke of th? curve are normal. The slow decline in the concentration of the dye is an indication of a large left to right shunt.

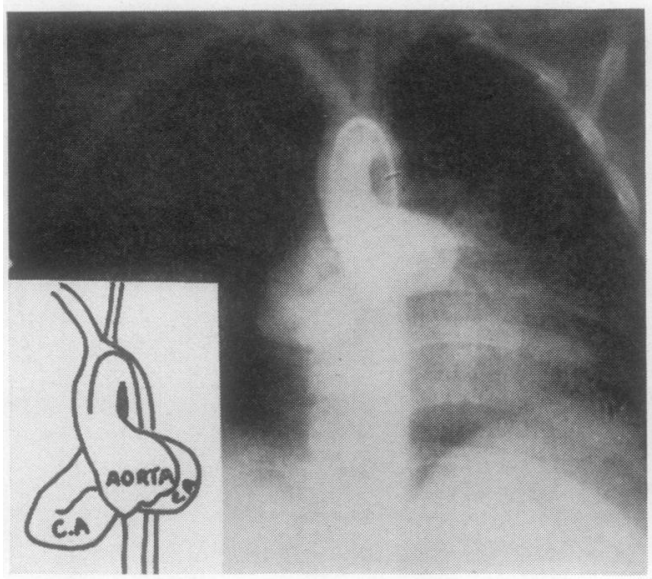

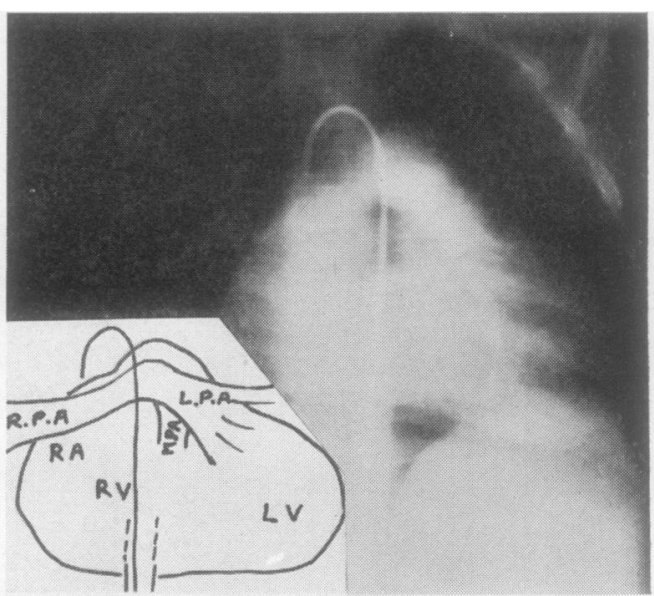

FIG. 7

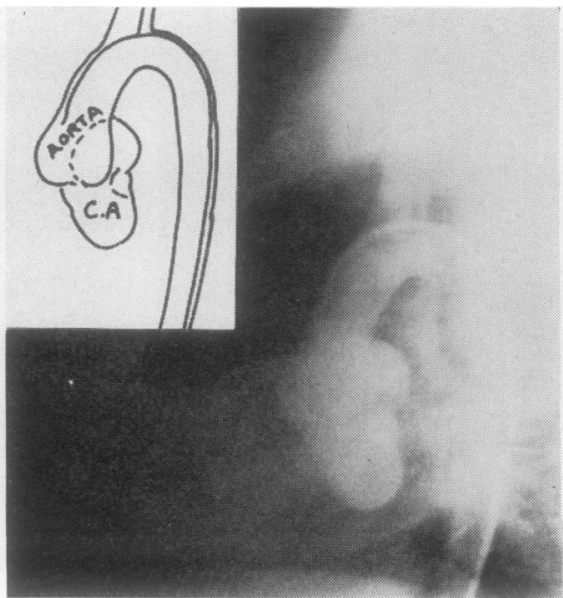

FIG. 6 
addition there was a wide tortuous vessel running posteriorly and to the right to enter the right auricle. A considerable quantity of radio-opaque medium passed through this abnormal vessel and successively outlined the right auricle, right ventricle, and pulmonary artery (Fig. 7). The right coronary artery was normal.

CASE 3 (M.L.) A woman aged 67 was referred for assessment of a murmur discovered incidentally during investigation of abdominal symptoms.

She was of slender build and had a blood pressure of $200 / 90 \mathrm{~mm}$. Hg. She was in sinus rhythm. The apex was in the fifth left intercostal space just outside the mid-clavicular line and there was no thrill.

The heart sounds were normal. There was a highpitched continuous murmur maximal in the third left intercostal space close to the sternum.

The electrocardiogram was normal. A chest radiograph showed the aorta unfolded and the heart a little enlarged to the left. The lung fields were normal (Fig. 8). Right heart catheterization and retrograde aortography were performed as in the previous cases. Pressures were normal in the right heart being 20/5, $20 / 2$, and $2 /-2 \mathrm{~mm}$. $\mathrm{Hg}$ in the pulmonary artery, right ventricle, and right atrium respectively. Blood oxygen saturation values were: superior vena cava, $66 \%$; inferior vena cava, $70 \%$; right atrium, $64 \%$; right ventricle, $65 \%$; pulmonary artery, $71 \%$, showing a left to right shunt into the pulmonary artery. A dye curve recorded from the ear after the injection of Coomassie blue into the pulmonary artery confirmed the existence of a small left to right shunt.

Aortography (Fig. 9) showed a tortuous dilated branch of the right coronary artery joining the pulmonary artery. The coronary artery continued with a normal calibre beyond the fistula. Opacification of the pulmonary artery through the fistula is best seen in the lateral view.

\section{DISCUSSION}

The majority of patients who have been diagnosed in life as suffering from this rare anomaly have only been recognized when thoracotomy has been undertaken for closure of a supposed patent ductus arteriosus. The literature contains at least 15 examples diagnosed at operation. One of these patients described by Walther, Starkey, Zervopolus, and Gibbons (1957) was later studied by cardiac catheterization, and another patient reported by Neill and Mounsey (1958) had an angiogram.

By contrast we have found only 10 cases diagnosed by angiography. A right-sided injection was used in four of these (Gasul, Arcilla, Fell, Lynfield, Bicoff, and Luan, 1960, cases 2 and 5; Neill and Mounsey, 1958 ; Bosher, Vasli, McCue, and Belter, 1959), but in other instances this

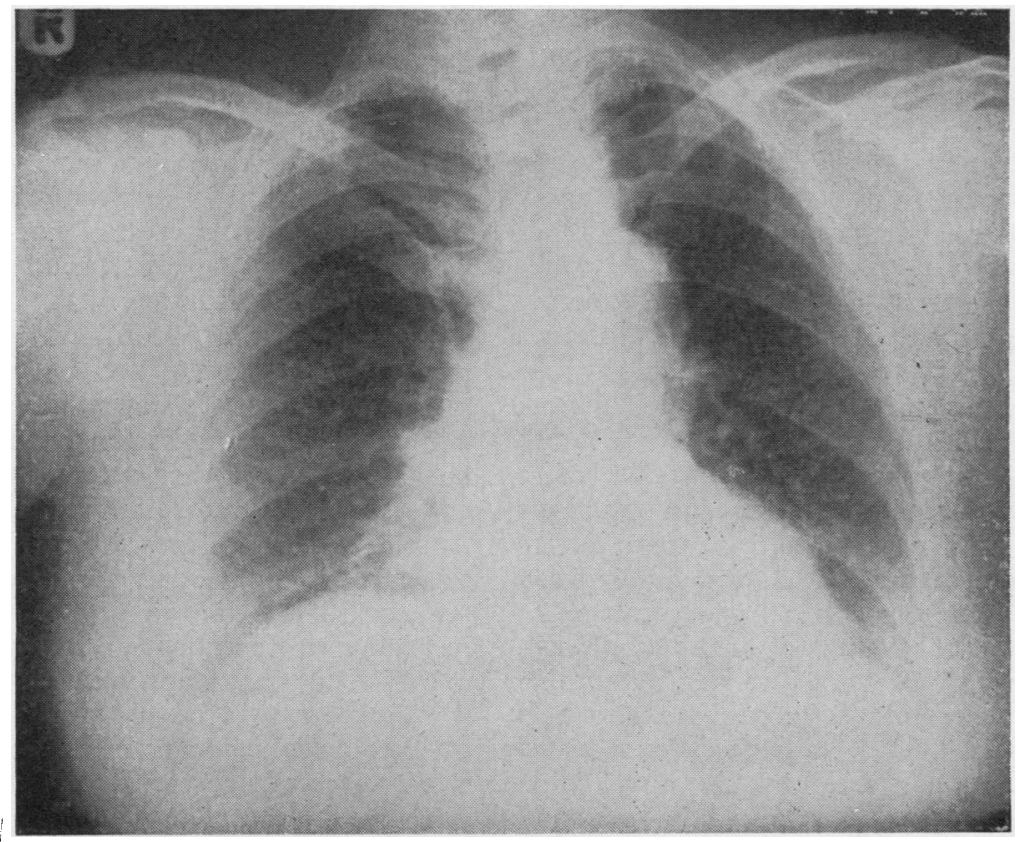

FIG. 8. Case 3. Chest radiograph shows the aorta is unfolded and the heart a little enlarged to the left. The pulmonery vessels are normal. 

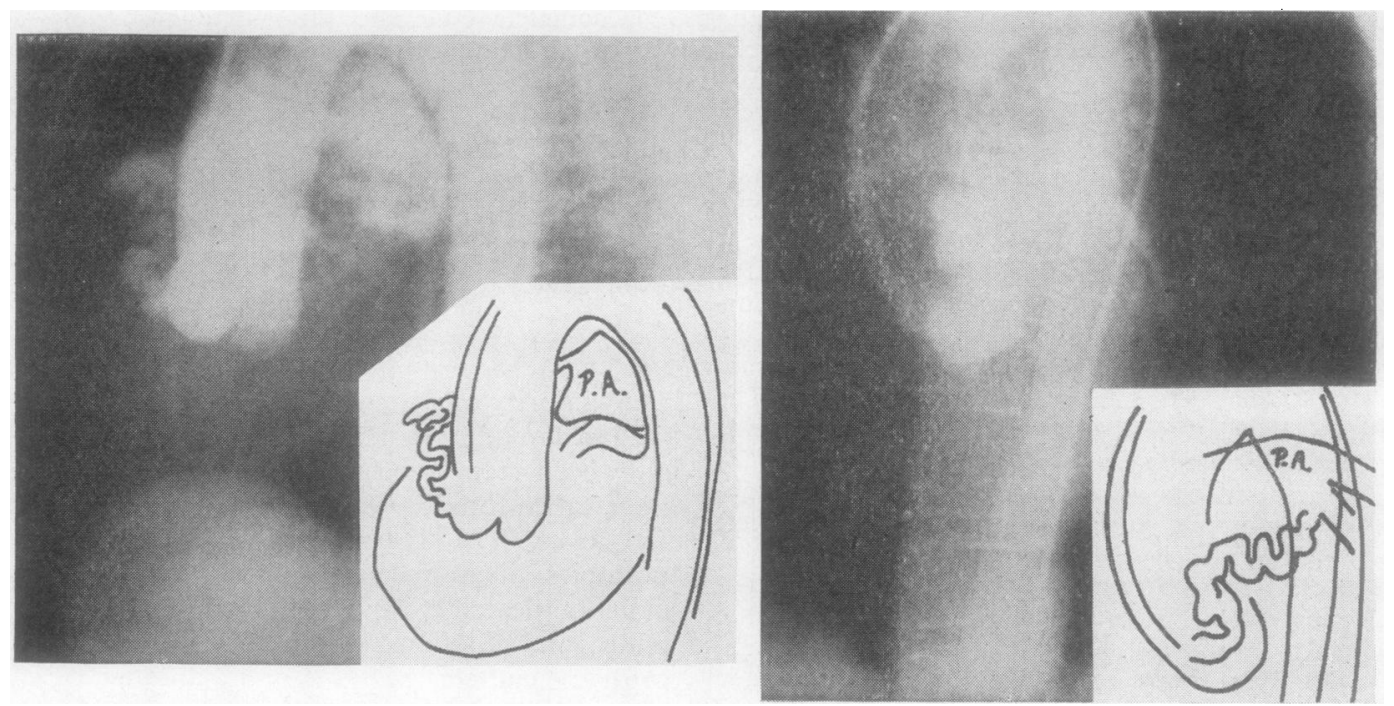

FIG. 9. Case 3. Retrograde thoracic aortogram. Lateral and antero-posterior films. The right coronary artery gives $\vec{\oplus}$ rise to a large abnormal branch which runs upwards and then backwards to the left and communicates with the pulmonary artery.

technique has failed to demonstrate the lesion because of dilution of the medium and superimposition of shadows of various cardiac chambers.

Retrograde aortography was used in the other six successful investigations (Neufeld, Lester, Adams, Anderson, Lillehei, and Edwards, 1961, cases 1-3; Munkner, Petersen, and Vesterdal, 1958 ; Bosher et al., 1959 ; and Gasul et al., 1960, case 4). In retrospect retrograde aortography was regarded as diagnostic in case 3 of Gasul et al. although it was misinterpreted prior to thoracotomy.

Cardiac catheterization has yielded mainly haemodynamic information, but in one instance (Walther et al., 1957, case 1) the diagnosis was established by this means alone, and Davison, McCracken, and Mcllveen (1955) were able to eliminate a patent ductus as the cause of a continuous murmur although the full diagnosis was not made until necropsy. In 20 cases left to right shunts were demonstrated, varying between $25 \%$ and $60 \%$ expressed as the percentage of pulmonary flow consisting of shunted blood. Pulmonary artery systolic pressure was $50 \mathrm{~mm}$. $\mathrm{Hg}$ or less in all except Bosher's patient, who also had a large patent ductus, and the cases reported by Neill and Mounsey and by Davison et al., where the patients were aged 56 and 58 years and had been in congestive heart failure. These findings are in keeping with the usual clinical history of a symptomless murmur dating from childhood, an important contrast to the history obtained in cases of rupture of an aneurysm of an aortic sinus into the right side of the heart, which is among the conditions to consider in differential diagnosis.

When considering the need for closure of a coronary arterio-venous fistula anatomical and pathological features are relevant. The abnormal vessel is invariably part of a normally distributed coronary artery with branches of normal size beyond the point of entry of the fistula into the right side of the heart. It is consequently possible to ligate only the distal end of the fistulous communication. Rupture of the dilated vessel does not seem to be a hazard, which is fortunate as distal ligation could not be expected to give 9 protection against this event. Bacterial infection is $\rightarrow$ a hypothetical complication listed by many authors but only in Gross' case, referred to by Paul, Sweet, N and White (1949), is it likely to have occurred. Another theoretical possibility is ischaemia of the myocardium in the territory supplied by normal $\omega$ branches of the abnormal vessel because of lower resistance to flow through the fistula. No instance 0 in which this has happened has been found, and $\mathbb{D}$ it is noteworthy that in Davison's case there was $\stackrel{?}{+}$ no histological evidence of ischaemia despite death at 58 years of age from congestive heart failure. This is in complete contrast with the severe ischaemic fibrosis and fatal cardiac failure in $\stackrel{\mathbb{D}}{\varrho}$ infancy brought about by the superficially 
analogous condition in which the left coronary artery arises from the pulmonary artery.

In our opinion, the risks of rupture, infection, and ischaemia are slight and do not constitute an indication for surgery, which is to be considered solely in terms of elimination of the shunt. Thoracotomy has been recorded in 25 cases, detailed by Gasul et al. (1960) together with the reports of Kay and Morris (1960) and Neufeld et al. (1961). The fistula was repaired in 22, the only reported death being that of a child who also had a large patent ductus arteriosus (Bosher et al., 1959). Nevertheless the operation may be one of some difficulty and hazard, and we have not so far felt justified in recommending it to any of our patients, although it may eventually be required in case 2 . Case 3 , who appears to be the oldest patient so far diagnosed in life, demonstrates no cardiovascular embarrassment and illustrates that a small fistula may be entirely benign.

\section{SUMMARY}

Three patients with a congenital fistula from a coronary artery to the right side of the heart are described.
Diagnosis was proved by retrograde aortography and the haemodynamic disturbance assessed by cardiac catheterization.

The possible consequences are discussed briefly in relation to operative treatment.

It is concluded that surgery is to be considered solely in terms of the size of the shunt.

We wish to thank Dr. D. Laing for allowing us to investigate case 3 .

\section{REFERENCES}

Bosher, L. H., Vasli, S., McCue, C. M., and Belter, L. F. (1959). Congenital coronary arteriovenous fistula associated with large patent ductus. Circulation, 20, 254.

Davison, P. H., McCracken, B. H., and McIlveen, D. J. (1955). Congenital coronary arterio-venous aneurysm. Brit. Heart J., $17,569$.

Gasul, B. M., Arcilla, R. A., Fell, E. H., Lynfield, J., Bicoff, J. P., and Luan, L. L. (1960). Congenital coronary arteriovenous fistula. Pediatrics, $25,531$.

Gross, R. E., quoted by Paul et al. (Personal communication).

Kay, H. B., and Morris, K. N. (1960). Congenital eoronary artery fistula to the right ventricle. Alfred Hosp. clin. Rep. (Melbourne), 10, 91

Munkner, T., Petersen, O., and Vesterdal, J. (1958). Congenital aneurysm of the coronary artery with an arteriovenous fistula. Acta radiol. (Stockh.), 50, 333.

Neill, C., and Mounsey, P. (1958). Auscultation in patent ductus arteriosus. Brit. Heart J., $20,61$.

Neufeld, H. N. Lester, R. G. Adams, P., Anderson, R. C. Lillehei, C. W., and Edwards, J. E. (1961). Congenital communication of a coronary artery with a cardiac chamber or the pulmonary trunk (coronary artery fistula). Circulation, $24,171$.

Paul, O., Sweet, R. H., and White, P. D. (1949). Coronary arteriovenous fistula. Amer. Heart J., 37, 441 .

Walther, R. J., Starkey, G. W. B., Zervopolus, E., and Gibbons, G. A. (1957). Coronary arteriovenous fistula. Amer. J. Med., 22, 213. 